\title{
Associação do fluxo sanguíneo periférico e capacidade funcional em amputados de membro inferior
}

Association of peripheral blood flow and functional capacity in lower limb amputees

Cássia da Luz Goulart ${ }^{1}$, Ana Paula Dattein Peiter ${ }^{1}$, Eduardo Bugs Eichelberger ${ }^{1}$, Rafael

Kniphoff da Silva ${ }^{1}$, Angela Cristina Ferreira da Silva ${ }^{1}$, Andréa Lúcia Gonçalves da

Siva $^{1}$

${ }^{1}$ Universidade de Santa Cruz do Sul, Santa Cruz do Sul/RS, Brasil.

Submissão: 11/08/2016

Aceite: 15/09/2016

andreag@unisc.br

\section{RESUMO}

Justificativa e Objetivo: A alteração do fluxo sanguíneo periférico (FSP) é fator de risco para diminuição da atividade física e amputação de membro inferior (MI). Avaliar a associação entre o FSP e a capacidade funcional de amputados traumáticos e não traumáticos de MI. Métodos: Estudo transversal realizado com 15 amputados de MI, usuários do Serviço de Reabilitação Física. Os pacientes foram avaliados pelo Índice Tornozelo Braquial (ITB), em decúbito dorsal, utilizando-se um esfigmomanômetro em membros superiores e MI preservado, para aferição da pressão arterial sistólica (com doppler vascular portátil) e cálculo do ITB. O Duke Activity Status Index (DASI) foi utilizado para avaliar atividades de vida diárias. Os pacientes foram alocados em dois grupos de amputados: não traumática $(\mathrm{GAnT}=6)$ e traumática $(\mathrm{GAT}=9)$. A análise estatística foi realizada pelo teste t de Student e correlação de Spearman para verificar associação entre as variáveis. Resultados: GAnT demonstrou predominância de amputação à esquerdo $(\mathrm{n}=3)$, mulheres $(\mathrm{n}=4)$, idade média $59,0 \pm 17,4$ anos, Índice de Massa Corporal- IMC $35,5 \pm 7,3 \mathrm{Kg} / \mathrm{m}^{2}$, DASI: $20,7 \pm 10,5$ e classificação do ITB em normal $(n=4)$ e DAP $(n=2)$. No GAT houve predominância de amputação à esquerdo $(\mathrm{n}=7)$, homens $(\mathrm{n}=9)$, idade $50,4 \pm 17,4$ anos, IMC $24,8 \pm 5,1 \mathrm{Kg} / \mathrm{m}^{2}$, DASI: $24,2 \pm 8,1 \mathrm{e}$ classificação do ITB em normal $(n=8)$ e ITB limítrofe $(n=1)$. O ITB geral diferiu significativamente entre GAnT e GAT $[0,93 \pm 0,17$ vs $1,11 \pm 0,12, p=0,03]$ e houve correlação positiva entre ITB e DASI no GAnT $(r=0,85 ; p=0,03)$. Conclusão: GAnT apresentam valores reduzidos de ITB e pior fluxo sanguíneo periférico que interfere diretamente na sua capacidade funcional.

DESCRITORES: Doença Arterial Periférica. Membro Inferior. Atividade Física.

\section{ABSTRACT}

Background and Aim: The change of peripheral blood flow (PBF) is a risk factor for decreased physical activity and amputation of lower limb (LL). Objectives: To evaluate the association between PBF and functional capacity of the LL amputees for traumatic 
and non-traumatic reason. Methods: Cross-sectional study with 15 amputees of the Physical Rehabilitation Service. Patients were assessed by Ankle Brachial Index (ABI) at rest in the supine position using a sphygmomanometer in the upper limbs and LL preserved for measurement of systolic blood pressure with Doppler vascular portable and to calculate the ABI. The Duke Activity Status Index (DASI) was used for evaluates daily life activities. Patients were allocated in two groups: non traumatic amputation $(\mathrm{GNTa}=6)$ and traumatic amputation $(\mathrm{GTa}=9)$. The analysis was performed by Student's t test and Spearman correlation to verify the association between variables. Results: In GNTa there was predominance of left amputation $(n=3)$, women $(\mathrm{n}=4)$, mean age $59.0 \pm 17.4$ years, Body Mass Index -BMI $35.5 \pm 7.3 \mathrm{~kg} / \mathrm{m}^{2}$, DASI: $20.7 \pm 10.5$ and classification of ABI in normal $(n=4)$ and DAP $(n=2)$. In G2 there was a predominance of left amputation $(n=7)$, men $(n=9)$, age $50.4 \pm 17.4$ years, BMI: $24.8 \pm 5.1 \mathrm{~kg} / \mathrm{m}^{2}$, DASI: $24.2 \pm 8.1$ and classification of $\mathrm{ABI}$ in normal $(\mathrm{n}=8)$ and $\mathrm{ABI}$ borderline $(n=1)$. The general ABI was difference between GNTa and GTa $[0.93 \pm 0.17$ vs $1.11 \pm 0.12, \mathrm{p}=0.03$ ] and positive correlation was found between ABI and DASI in GNTa ( $r=0.85 ; p=0.03$ ). Conclusion: GNTa have reduced levels of ABI and worse peripheral blood flow, and this affects your functional capacity.

KEYWORDS: Peripheral Arterial Disease. Lower Extremity. Motor Activity.

\section{INTRODUÇÃO}

A amputação, caracterizada pela remoção cirúrgica ou traumática de algum segmento, está entre as principais causas de incapacidade da população mundial. ${ }^{1}$ Cerca de $80 \%$ de todas as amputações de membros inferiores resultam de doença arterial periférica e de complicações do Diabetes Mellitus, gerando deficiência, além de alterações físicas, psicológicas e sociais. ${ }^{2}$ Dentre estas alterações, a redução do nível de atividade física e da capacidade funcional destes indivíduos predispõe a obesidade e ao sedentarismo. ${ }^{3}$

A obesidade e a inatividade física são reconhecidas como importantes fatores para o desenvolvimento de doenças cardiovasculares. ${ }^{4}$ A Doença Arterial Periférica (DAP) é uma das doenças cardiovasculares mais debilitantes, sendo na maioria dos casos diagnosticada em estágios mais avançados da doença, quando esta se torna sintomática. ${ }^{5} \mathrm{O}$ Índice Tornozelo-Braquial (ITB) é um método não invasivo e de simples realização, fornecendo importantes informações acerca de diagnóstico para a DAP. Além disso, valores abaixo ou acima dos limites de normalidade têm sido associados ao risco de mortalidade por doenças cardiovasculares. ${ }^{6} \mathrm{Em}$ pacientes amputados, a análise do ITB se torna importante para avaliar a possibilidade de uma amputação no membro contralateral, sendo este um risco sempre presente. ${ }^{2}$

Neste sentido, nós hipotetizamos que os amputados por motivo não traumático possuem uma capacidade funcional e fluxo sanguíneo reduzido quando comparados aos 
amputados traumáticos. Objetivamos assim, avaliar se há associação entre o fluxo sanguíneo periférico e a capacidade funcional de amputados de membro inferior (MI) por motivo traumático e não traumático.

\section{MÉTODOS}

\section{Delineamento do estudo}

Estudo de delineamento transversal, com amostragem de conveniência não probabilística, realizado junto ao Serviço de Reabilitação Física (SRFis) da Universidade de Santa Cruz do Sul (UNISC), estabelecido na Clínica FisioUNISC, no município de Santa Cruz do Sul - RS. O SRFis é o centro de referência para $8^{\mathrm{a}}, 13^{\mathrm{a}} \mathrm{e}$ $16^{\text {a }}$ Coordenadorias Regionais de Saúde na dispensação de órteses, próteses e meios auxiliares de locomoção, financiados pelo Sistema Único de Saúde através do Ministério da Saúde e do Governo Estadual do Rio Grande do Sul desde o ano de 2009. Esta pesquisa foi devidamente aprovada pelo Comitê de Ética em Pesquisa da UNISC com protocolo $\mathrm{n}^{\mathrm{o}} 54494916.7 .0000 .5343$ e todos os sujeitos envolvidos consentiram e assinaram um termo de consentimento livre e esclarecido.

\section{Sujeitos da pesquisa}

Foram incluídos na pesquisa 15 amputados unilaterais de membro inferior agrupados em: amputação não traumática $(\mathrm{GAnT}=6)$ e amputação traumática $(\mathrm{GAT}=9)$. O agrupamento ocorreu independente do nível e tempo de amputação, do sexo, em adultos com idade superior a 18 anos, com boa cognição e que aceitarem voluntariamente participar desta pesquisa. Sendo excluídos amputados com déficit neurológico ou ortopédico, que apresentem algum tipo de lesão de pele que impedissem de cumprir o protocolo da pesquisa.

\section{Mensurações}

\section{Duke Activity Status Index}

Composto por 12 itens envolvendo cuidados pessoais, deambulação, tarefas domésticas, atividade sexual e atividade recreativa, em que cada item representa uma pontuação baseada proporcionalmente ao gasto metabólico de cada atividade. Também é utilizado para predizer capacidade funcional e consumo de oxigênio $\left(\mathrm{VO}_{2}\right)$ sem a necessidade de ser realizado o teste cardiorrespiratório máximo. $\mathrm{O} \mathrm{VO}_{2}\left(\mathrm{~mL} . \mathrm{kg}^{-1}\right.$. 
$\min ^{-1}$ ) é estimado por meio da seguinte equação de regressão linear múltipla: $\mathrm{VO}_{2}=$ $0,43 \times \mathrm{DASI}+9,6$. $^{7}$

\section{Índice Tornozelo Braquial}

O ITB foi aferido com os indivíduos em decúbito dorsal conforme recomendação da American Heart Association. Realizada a aferição da PAS das artérias braquiais, pediosa e tibial posterior bilateralmente através de doppler vascular portátil (MEDPEJ® - modelo 2001, Brasil) com esfigmomanômetro posicionado 03 centímetros acima da fossa cubital nos membros superiores e 03 centímetros acima do maléolo medial em membro inferior (MI). Após a aferição das medidas, foi realizado cálculo dividindo-se o maior valor entre os MI pelo maior valor entre os membros superiores para o cálculo do ITB geral. ${ }^{8}$ Os amputados avaliados foram classificados conforme Kim, Wattanakit e Gornik (2012), sendo que um índice de 1.00 a 1.40 é considerado normal, 0.91 a 0.99 limítrofes e menores que 0.90 DAP. ${ }^{9}$

\section{Análise Estatística}

Os dados foram inseridos e analisados no programa estatístico SPSS versão 20.0 (Systat Software, Alemanha). De acordo com a natureza da distribuição das variáveis utilizou-se as medidas de tendência central e de dispersão para apresentação dos resultados: média e desvio padrão (paramétricas) ou mediana com intervalo mínimo e máximo (não-paramétricas). Realizada comparações entre os grupos através do teste $\mathrm{t}$ de Student, para análises das variáveis categóricas o teste de qui-quadrado e associações as pelo Coeficiente de Correlação de Pearson. Um modelo de regressão linear simples foi utilizado para avaliar a influência da capacidade funcional sobre o ITB. Os resíduos foram avaliados segundo as suposições de normalidade, variância constante e independência. Foi considerado significativo um $p<0,05$.

\section{RESULTADOS}

As características clínicas dos sujeitos estudados estão descritas na Tabela 1. É possível observar características semelhantes entre os grupos de amputados exceto para a predominância do sexo masculino e maior ITB no grupo de amputados traumáticos. 
Tabela 1 - Características clínicas dos sujeitos amputados.

\begin{tabular}{|c|c|c|c|}
\hline Variáveis & GaNT $(n=6)$ & GT $(n=9)$ & $P$ \\
\hline \multicolumn{4}{|l|}{$\mathrm{Sexo}^{\alpha}, \mathrm{n}(\%)$} \\
\hline Masculino & $2(33,3)$ & $9(100,0)$ & 0,03 \\
\hline Feminino & $4(66,7)$ & - & - \\
\hline Idade (anos) $^{\bullet}$ & $59,0 \pm 10,7$ & $50,4 \pm 17,4$ & 0,22 \\
\hline $\operatorname{IMC}\left(\mathrm{Kg} / \mathrm{m}^{2}\right)^{\bullet}$ & $35,5 \pm 7,3$ & $24,8 \pm 5,1$ & 0,31 \\
\hline \multicolumn{4}{|c|}{ Nível da amputação ${ }^{\alpha}, \mathrm{n}(\%)$} \\
\hline Transtibial & $1(16,7)$ & $1(11,1)$ & 1,00 \\
\hline Transfemoral & $5(83,3)$ & $8(88,9)$ & 0,40 \\
\hline \multicolumn{4}{|c|}{ Classificação IMC ${ }^{\alpha}, \mathrm{n}(\%)$} \\
\hline Normal & - & $5(55,6)$ & - \\
\hline Sobrepeso & $2(33,3)$ & $2(22,2)$ & 1,00 \\
\hline Obesidade & $4(66,7)$ & $2(22,2)$ & 0,41 \\
\hline $\mathrm{RCQ}(\mathrm{cm})^{\circ}$ & $0,9 \pm 0,0$ & $0,8 \pm 0,0$ & 0,09 \\
\hline \multicolumn{4}{|c|}{ Classificação $\mathrm{RCQ}^{\alpha}, \mathrm{n}(\%)$} \\
\hline Baixo & - & $5(55,6)$ & - \\
\hline Moderado & - & $2(22,2)$ & - \\
\hline Alto & $2(33,3)$ & $2(22,2)$ & 1,00 \\
\hline Muito Alto & $4(66,7)$ & & - \\
\hline $\operatorname{ITB}_{(\text {geral })^{\bullet}}$ & $0,9 \pm 0,1$ & $1,1 \pm 0$ & 0,03 \\
\hline \multicolumn{4}{|c|}{ Classificação ITB ${ }^{\alpha}, \mathrm{n}(\%)$} \\
\hline Normal & $4(66,7)$ & $8(88,9)$ & 0,24 \\
\hline Limítrofe & - & $1(11,1)$ & - \\
\hline DAP & $2(22,2)$ & - & - \\
\hline DASI $^{\circ}$ & $20,7 \pm 10,5$ & $24,2 \pm 8,1$ & 0,50 \\
\hline
\end{tabular}

Dados expressos em média \pm desvio padrão e frequência; ${ }^{\alpha}$ Qui-quadrado; ${ }^{\circ}$ Teste t de Student independente; GaNT: grupo não traumático; GT: grupo traumático; IMC: Índice de Massa Corporal; RCQ: Relação Cintura Quadril; ITB: Índice Tornozelo Braquial; DAP: Doença arterial periférica; DASI: Duke Activity Status Index.

Na Figura 1 estão demonstradas as correlações entre DASI e o ITB, onde encontramos correlação significativa e forte no GaNT, demonstrando assim, que quanto maior é o ITB melhor é a capacidade funcional destes amputados.
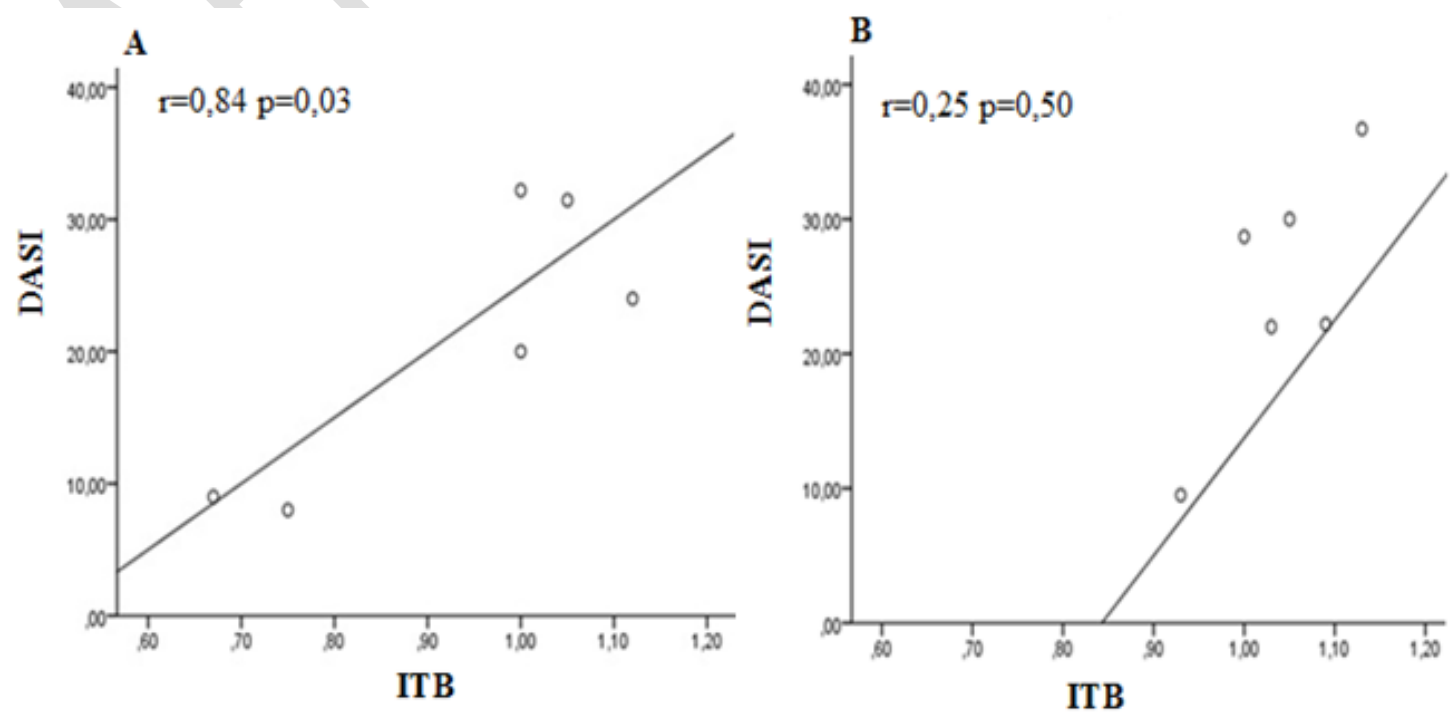
Figura 1 - Associação entre o fluxo sanguíneo periférico e a capacidade funcional. A: Grupo amputados não traumático; DASI: Duke Activity Status Index; ITB: Índice tornozelo braquial; B: Grupo traumático. Realizada correlação de Pearson.

Aplicamos um modelo de regressão linear simples e identificamos que o fluxo sanguíneo periférico, explica $84 \%$ do resultado da capacidade funcional no GaNT.

Tabela 2 - Análise de regressão linear simples para predizer a capacidade funcional, a partir do ITB no GaNT.

\begin{tabular}{lcc}
\hline Variáveis & Coeficiente B & $p$-valor \\
\hline Constante & 0,66 & $<0,001$ \\
ITB & 0,01 & 0,034
\end{tabular}

GaNT: Grupo amputado Não Traumático; ITB: Índice Tornozelo Braquial; $\overline{\mathrm{R}}_{\text {ajustado }}=$ 0,$84 ; \mathrm{F}=10,10(p=0,03)$.

\section{DISCUSSÃO}

Os achados primários do presente estudo mostraram que os sujeitos amputados por motivo não traumático apresentaram uma associação forte entre o fluxo sanguíneo periférico e a capacidade funcional, demonstrada também pela análise de regressão linear. Estes achados podem ter particular relevância sobre as futuras implicações da amputação na capacidade funcional destes sujeitos e sua relação com o fluxo sanguíneo periférico durante as atividades de vida diária.

A amputação pode resultar em uma redução da mobilidade, afetando os sistemas musculoesquelético, cardiovascular e respiratório. Além disso, existem evidências que a imobilidade interfere também no sistema imunológico, diminuindo a resistência contra doenças infeciosas. ${ }^{2}$ Sendo assim, a redução do nível de atividade física pode estar relacionada com a capacidade funcional cardiovascular, que varia de acordo com a idade, nível de amputação, etiologia da amputação, presença de comorbidades, e utilização de próteses. ${ }^{10}$

Podemos ressaltar que em nosso estudo, houve predomínio de amputação transfemoral e por causa traumática e, segundo Esposito et al. (2014), os amputados por causa traumática necessitam de um gasto metabólico menor quando comparado aos amputados de etiologia vascular, que por sua vez apresentam pior aptidão física, uma vez que suas amputações são resultantes de alterações nas condições metabólicas, como o Diabetes Mellitus ou a DAP. ${ }^{11}$

A retirada parcial ou total de um membro, interfere diretamente na capacidade funcional do individuo podendo também alterar o fluxo sanguíneo do membro inferior e repercutir assim na hemodinâmica cardiovascular. ${ }^{1,12,13}$ A DAP, resultante de um 
processo aterosclerótico, que afeta as artérias coronárias e promove a oclusão de artérias dos membros inferiores, é amplamente variável ao redor do mundo, e no Brasil ainda há poucos registros na literatura atual, sendo que sua identificação é de fundamental importância já que esta aumenta o risco de evento cardiovascular e possível amputação contralateral. $^{10,12}$ Mesmo conhecendo pouco sobre a sua prevalência em sujeitos amputados, em nosso estudo, encontramos uma pequena frequência de DAP, porém foi possível identificar que as alterações no fluxo sanguíneo interferiram $84 \%$ na capacidade funcional dos amputados avaliados.

Sendo assim, concluímos que os amputados por motivos não traumáticos possuem uma capacidade funcional reduzida e que está se encontra diretamente associada ao pior fluxo sanguíneo periférico.

\section{Agradecimento}

Ao Serviço de Reabilitação Física e Grupo de pesquisa "Reabilitação em Saúde e suas Interfaces" pelo suporte financeiro.

\section{REFERÊNCIAS}

1. Knezevic A, Salamon T, Milankov M, et al. Assessment of quality of life in patients after lower limb amputation. Medicinski Pregled 2015; 68 (3): 103108.DOI: http://dx.doi.org/10.2298/MPNS1504103K.

2. Stefancic M, Presern-Strukel JM, Vidmar G, et al. Immunological status in patients with lower limb amputation due to peripheral arterial disease before and after comprehensive rehabilitation. Coll Antropol 2015; 39 (1): 127-130.

3. Littman AJ, Thompson ML, Arterburn DE, et al. Lower-limb amputation and body weight changes in men. Journal of Rehabilitation Research \& Development 2015; 52 (2): 159-170.

4. Gus I, Ribeiro RA, Kato S, et al. Variações na prevalência dos fatores de risco para doença arterial coronariana no Rio Grande do Sul: uma análise comparativa entre 2002-2014. Arq Bras Cardiol 2015; 105 (6): 573-579.

5. Yeboah K, Puplampu P, Yorke E, et al. Body composition and ankle-brachial index in Ghanaians with asymptomatic peripheral arterial disease in a tertiary hospital. BMC Obesity 2016; 27(3): 1-7. 
6. Santos VP, Alves CAS, Fidelis RJR, et al. Estudo comparativo do Índice Tornozelo-Braquial em diabéticos e não diabéticos com isquemia crítica. J Vasc Bras 2015; 14 (4): 305-310.

7. Maranhão NGA, Lourenço PM, Farinatti PT. Prediction of aerobic fitness without stress testing and applicability to epidemiological studies: a systematic review. Cad Saude Publica 2004; 20 (1): 48-56.

8. Aboyans V, Criqui MH, Abraham P, et al. Measurement and interpretation of the ankle-brachial index: A scientific statement from the Americam Heart Association. Circulation J Am Heart Assoc 2012; 126 (24): 2890-2909.

9. Kim EH, Wattanakit K, Gornik HL. Using the ankle-brachial index to diagnose peripheral artery disease and assess cardiovascular risk. Cleveland Clinic J Med 2012; 79 (9): 651-61.

10. Lin MS, Hsu KS, Chen YJ, et al. Prevalence and Risk Factors of Asymptomatic Peripheral Arterial Disease in Patients with COPD in Taiwan. Plos One 2013; 8(5):e64714. DOI: http://dx.doi.org/10.1371/journal.pone.0064714

11. Esposito ER, Rodriguez KM, Rabago CA, et al. Does unilateral transtibial amputation lead to greater metabolic demand during walking? J Rehab Research Develop 2014; 51 (8): 1287-1296.

12. Sun KS, Lin MS, Chen YJ, et al. Is asymptomatic peripheral arterial disease associated with walking endurance in patients with COPD?. Int J Chron Obstruct Pulmon Dis 2015; 10 1487-1492.

13. Chen R, Wanbing H, Zhang K, et al. Airflow obstruction was associated with elevation of brachial-ankle pulse wave velocity but not ankle-brachial index in aged patients with chronic obstructive pulmonary disease. Atherosclerosis 2015; 242 (1):135-140. 